\title{
Measurements of Nonlinear Harmonic Generation at the Advanced Photon Source's SASE FEL*
}

\author{
S.G. Biedron ${ }^{\star 1}$, R.J. Dejus, Z. Huang, S. V. Milton, V. Sajaev, W. Berg, M. Borland, \\ P. K. Den Hartog, M. Erdmann, W.M. Fawley ${ }^{2}$, H.P. Freund ${ }^{3}$, E. Gluskin, K.-J. Kim, \\ J. W. Lewellen, Y. Li, A. H. Lumpkin, E. R. Moog, A. Nassiri, G. Wiemerslage, and B. X. Yang \\ Advanced Photon Source, Argonne National Laboratory, Argonne, IL 60439, USA \\ 1 and MAX-Laboratory, University of Lund, Sweden 22100 \\ ${ }^{2}$ Lawrence Berkeley National Laboratory, Berkeley, CA 94720, US A \\ ${ }^{3}$ Science Applications International Corporation, McLean, VA 22102, US A
}

\begin{abstract}
* The work of the Argonne authors is supported by the U.S. Department of Energy, Office of Basic Energy Sciences under Contract No. W-31-109-ENG-38. The activity of W.M. Fawley is supported at Lawrence Berkeley National Laboratory by the U.S. Department of Energy under Contract No. DE-AC03-76SF00098. The activity and computational work for H.P. Freund is supported by Science Applications International Corporation's Advanced Technology Group under IR\&D subproject 01-0060-73-0890-000. Computational resources were provided in part by the National Energy Research Supercomputer Center (NERSC).
\end{abstract}

PACS Codes: 41.60, 42.65.Ky, 52.59.-f

Keywords: Free-Electron Lasers, Harmonic Generation, Frequency Conversion, Intense Particle Beams and Radiation Sources

- Corresponding Author: APS, ANL 9700 South Cass Avenue, Argonne, Illinois 60439, USA; email address: biedron@aps.anl.gov, Telephone: 630252 1162, Facsimile: 6302525703

\section{Abstract}

SASE saturation was recently achieved at the Advanced Photon Source's SASE FEL in the low-energy undulator test line (LEUTL) at $530 \mathrm{~nm}$ and $385 \mathrm{~nm}$. The electron beam microbunching becomes more and more prominent until saturation is achieved. This bunching causes nonlinear harmonic emission that extends the usefulness of a SASE system in achieving shorter FEL wavelengths for the same electron beam energy. We have investigated the intensity of the fundamental and second-harmonic undulator radiation as a function of distance along the undulator line and present the experimental results and compare them to numerical simulations. In addition, we have measured the single-shot second harmonic spectra as well as the simultaneous fundamental and second harmonic spectra and present the experimental results.

\section{Introduction}

The Advanced Photon Source's (APS's) SASE FEL in the low-energy undulator test line

(LEUTL) demonstrated exponential gain to saturation [1]. This was shown for the cases of a fundamental radiation wavelength of $530 \mathrm{~nm}$ and also $385 \mathrm{~nm}$. 
Microbunching occurs during the FEL process. This bunching at the fundamental is temporally spiky in nature and when Fourier decomposed, shows significant power growth at higher harmonics. These nonlinear harmonics grow due to the existence of the fundamental and, like the fundamental, experience gain to saturation, with gain lengths that vary as the inverse of their harmonic number [2-5]. These nonlinear harmonics and related harmonic schemes have been discussed extensively in the literature [2-15]. Experimentally, a number of related harmonic measurements have been recently conducted. These include the fundamental, second, and third nonlinear harmonics at the high-gain harmonic generation (HGHG) experiment at Brookhaven National Laboratory (BNL) [16]; nonlinear harmonic spectral measurements $[17,18]$ at the Institute of Scientific and Industrial Research in Osaka University, Japan; and nonlinear harmonic spectral and intensity measurements at the VISA (Visible to Infrared SASE FEL) experiment at BNL [19].

\section{Experimental Layout}

For each of the three experimental and simulated cases described below, the APS SASE FEL was operated at an electron beam energy of $217 \mathrm{MeV}$ corresponding to 530-nm fundamental radiation for a planar undulator with a 3.3-cm period length and a strength parameter, K, of 3.1. We also note that we operated the APS SASE FEL in the "short-pulse" mode when these measurements were obtained. This short (electron) pulse case occurs when the electron bunch length becomes comparable to or less than the slippage length $\mathrm{L}_{\mathrm{s}}=$ $\mathrm{N}_{\text {und }} \lambda_{\text {rad }}$, where $\mathrm{N}_{\text {und }}$ is the number of undulator periods and $\lambda_{\text {rad }}$ is the radiation wavelength. In this case, there is less interaction of the radiated energy back upon the electron beam. (At a wavelength of $530 \mathrm{~nm}$, the slippage length through nine undulators is $3.5 \times 10^{-4} \mathrm{~m}$, corresponding to $116 \mathrm{fs}$.) This effect increases the gain length and reduces the final output energy compared to the long pulse case. 
After each undulator segment in the LEUTL line, there exists an actuator with a mirror that may be inserted into the beamline to direct the undulator radiation (UR) through a series of filters in front of each CCD camera. The filters allow control of the measured radiation wavelength and intensity. Before the early March 2001 (experimental) "runs," the camera assemblies were capable of viewing down to $370 \mathrm{~nm}$. For the run of 8 March 2001, diagnostic stations 6 and 8 were upgraded to view down to $200 \mathrm{~nm}$. In addition, two solar blind filters, able to block radiation longer than $360 \mathrm{~nm}$ and shorter than $225 \mathrm{~nm}$, were installed on the filter wheels. By the 30 March 2001 run, stations 2 and 4 were also outfitted with these same upgrades. By the 16 August run, all stations had been upgraded. In all of these cases, however, the 530-nm radiation was readily detectable at every diagnostic station.

In addition to measuring the UR, we have the capability to measure the coherent transition radiation (CTR), a direct measure of the electron beam microbunching [20]. We do not, however, review these measurements here.

Measurement of the harmonic spectra at each diagnostic station was performed by redirecting the light using a second mirror mounted on a motorized flipper at each station toward a spectrometer [21] in the LEUTL end-station diagnostic room.

\section{Measurements and Simulations and their Analysis}

\section{A. First Experiments}

On 8 March 2001, we measured the fundamental $(530 \mathrm{~nm})$ UR intensities at all the stations and also the second harmonic (265 nm) UR intensities at stations 6 and 8. In addition, we measured the harmonic spectra at four stations $(5,6,7$, and 8 at $12 \mathrm{~m}, 14.4 \mathrm{~m}, 16.8 \mathrm{~m}, 19.2$ $\mathrm{m}$, respectively) using the end-station spectrometer. Table 1 lists the relevant electron beam parameters for these measurements. 
We have used the simulation code GINGER to simulate the expected performance of our system [22,23]. GINGER is able to simulate the short-pulse case described above, including start up from noise, but it cannot examine the energies of the nonlinear harmonic emission. It can, however, examine the fundamental energy and microbunching at the fundamental and nonlinear harmonics. For all the simulated cases examined here, we ran and then averaged four GINGER simulations to demonstrate, on average, the predicted SASE emission. We then employed an analytical model [5] that allowed us to use the bunching factors for the fundamental and second harmonic and the energy of the fundamental radiation from the GINGER simulations to obtain a theoretical estimate for the second harmonic energy. This model relates the second harmonic energy, $\mathrm{E}_{2}$, to the fundamental energy $\mathrm{E}_{1}$, and is given by

$$
\mathrm{E}_{2} \approx \mathrm{E}_{1}\left(\frac{\mathrm{K}}{\gamma_{0} \mathrm{k}_{\mathrm{u}} \sigma_{\mathrm{x}}}\right)^{2}\left(\frac{\mathrm{K}_{2}}{\mathrm{~K}_{1}}\right)^{2}\left(\frac{\mathrm{b}_{2}}{\mathrm{~b}_{1}}\right)^{2},
$$

where $\mathrm{K}$ is the undulator parameter, $\gamma_{0}$ is the relativistic factor of the electron beam, $\mathrm{k}_{\mathrm{u}}$ is the wavenumber of the undulator period, $\sigma_{\mathrm{x}}$ is the average rms electron beam size in the wiggling plane, $\mathrm{K}_{1}$ and $\mathrm{K}_{2}$ are the effective coupling strength factors due to the fundamental and second harmonic radiation, respectively, and $b_{1}$ and $b_{2}$ are the microbunching factors of the electron beam at the fundamental and second harmonic wavelengths, respectively. Note the effective coupling strengths $\mathrm{K}_{1}$ and $\mathrm{K}_{2}$ are expressed in terms of Bessel functions and are given by:

$$
\mathrm{K}_{\mathrm{h}}=\mathrm{K}(-1)^{(\mathrm{h}-1) / 2}\left[\mathrm{~J}_{(\mathrm{h}-1) / 2}(\mathrm{~h} \xi)-\mathrm{J}_{(\mathrm{h}+1) / 2}(\mathrm{~h} \xi)\right]
$$

for $\mathrm{h}=1,3,5, \ldots$ and by

$$
\mathrm{K}_{\mathrm{h}}=\mathrm{K}(-1)^{(\mathrm{h}-2) / 2} \mathrm{~J}_{\mathrm{h} / 2}^{\prime}(\mathrm{h} \xi)
$$

for $\mathrm{h}=2,4, \ldots$, where $\xi=\mathrm{K}^{2} /\left(4+2 \mathrm{~K}^{2}\right)$. 
Figure 1 shows the results of the GINGER simulation, the GINGER simulation results applied to the above-listed analytical method, and the measured UR radiation as obtained during the measurements of 8 March 2001. Note the measured gain curve at $530 \mathrm{~nm}$ for the UR showed saturation at and beyond station 6. (In all of the GINGER simulations discussed herein, we used an rms energy spread of $0.15 \%$. We also wish to mention that the effective wavelength bandwidth used in these GINGER simulations was $\pm 20 \%$.) Here, we have normalized the fundamental energy from GINGER and scaled the analytically found second harmonic energy to the experimental fundamental measurement at station $2(4.8 \mathrm{~m})$, where the SASE coherent emission should strongly dominate the broadband spontaneous emission. From simulation and the analytical theory, the averaged, predicted fundamental-tosecond-harmonic UR ratio is $\sim 1500$ at saturation and the average ratio of the fundamental to the second harmonic in the saturated regime from the two UV stations is 230 . The electron beam parameters were held constant during the gain scans (530 and $265 \mathrm{~nm}$ ), so the comparison of the intensities is particularly relevant. However, the variation in ratios from the two UV stations is large, and we cannot assign a reliable error estimate to these ratios; we merely note that the fluctuations are of the order of about $30 \%$.

On 8 March, we also used the end-station spectrometer to measure the single-shot second harmonic spectrum after undulators $5,6,7$, and $8(12 \mathrm{~m}, 14.4 \mathrm{~m}, 16.8 \mathrm{~m}, 19.2 \mathrm{~m}$, respectively). Note that these multiple, single-shot spectra at different stations were taken at different times, as there is no possibility to take them all simultaneously. Here, we show three representative single-shot spectra at each of these four stations in Figures 2 (a) - (d), respectively. From the fundamental gain scan, we know that the system is saturated for both the fundamental and higher harmonics at and beyond station 6. Note this is also consistent 
with the spectral narrowing observed at station 5 compared to station 6 , and then the spectral broadening, shift to the red, and appearance of sidebands at stations 7 and 8 are indicative of being in the region beyond FEL saturation. Since the transmissive losses are unknown for the optical transport line from the in-tunnel mirror to the end-station spectrometer, we are currently unable to compare spectral intensity levels with that from the CCD detectors used to quantify the emission. The average rms relative spectral widths of the second harmonic emission after stations $5,6,7,8$ as a function of distance are shown in Figure 3. Note the clear increase in the average rms relative spectral width once saturation has been reached. Finally, the absolute spectral widths of the single-shot spectra are not estimated quantitatively here as this matter is under further investigation.

\section{B. Second Experiments}

On 30 March 2001, for which we had a significantly higher peak electron beam current, the fundamental $(530 \mathrm{~nm})$ UR intensities were measured at every station, and the second harmonic $(265 \mathrm{~nm})$ UR intensities were measured at the even diagnostic stations. In addition, the simultaneous fundamental and second harmonic spectra were measured at stations $6,7,8$ and $9(14.4 \mathrm{~m}, 16.8 \mathrm{~m}, 19.2 \mathrm{~m}$, and $21.6 \mathrm{~m}$, respectively). The electron beam properties were measured after the 530-nm gain scan and then again after the 265-nm gain scan. These electron beam properties are listed in Table 2. Since the undulator radiation was measured for different electron beam conditions, these data sets cannot be directly compared with one another.

Figure 4 shows both the experimental $530-\mathrm{nm}$ gain scan results and those from the GINGER simulations corresponding to beam parameters in column 1 of Table 2 (530-nm gain scan). As is clearly seen, the fundamental scan saturates at station 6 , where $z=14.4 \mathrm{~m}$. 
The fundamental UR energy from GINGER is normalized to the third measured data point, where we are experimentally in the exponential gain regime. Here, we find the measured fundamental gain length of $0.74 \mathrm{~m}$, compared to a predicted $0.67 \mathrm{~m}$. The overall longitudinal offset of the measured and predicted curves and the increase in the gain length of the measured curve are perhaps due to a missteering at the entrance to the undulator.

Again, the fundamental energy and fundamental and second harmonic bunching factors resulting from simulations with GINGER were used to calculate the second harmonic energy. The measured 265-nm data and the estimated second harmonic energy from equation (1) are shown in Figure 5. The estimated second harmonic energy has been normalized to the second measured second harmonic data point (station 4) since the spontaneous radiation dominates the first measured data point (station 2). We have insufficient experimental data to determine the second harmonic gain length using these measured points. Note that the third data point lies well into saturation. We currently believe that the additional, higher than expected measured second harmonic point at $\mathrm{z}=5 \mathrm{~m}$ may be a result of the solar blind filter alignment that may lead to leakage of the fundamental radiation to the detector simultaneously.

For this data set, we also obtained simultaneous single-shot spectra for the fundamental and second harmonic emission using the end-station spectrometer by observing the radiation individually from undulators $6,7,8$, and 9. Typical spectra are presented in Figure 6 . It is interesting that while the fundamental spectrum is still dominated by a single mode, the second harmonic spectrum is quite rich, with four nearly equally spaced peaks of emission. From the fundamental and second harmonic gain scans, we assess again that we were near the point of saturation at a distance of $\sim 15 \mathrm{~m}$ (near station 5 ). We wish to mention that this 
was one of numerous single shots taken, and sometimes the spike structure between the fundamental and second harmonic are similar and sometimes they are not. This point is still under investigation.

\section{Third Experiments}

In August 2001, a third set of intensity measurements at $530 \mathrm{~nm}$ and $265 \mathrm{~nm}$ were made with data available at $265 \mathrm{~nm}$ from all diagnostic stations. The relevant electron beam parameters are listed in Table 3, while the reduced data are plotted in Figure 7, again with predictions from the GINGER code. The data point at station $7(16.8 \mathrm{~m})$ is suspect due to questions related to the alignment of the optical system and the solar blind filters in particular. The measured gain length of the fundamental is $0.75 \mathrm{~m}$ while that of the second harmonic is $0.55 \mathrm{~m}$; these should be considered upper limits due to the limited sampling frequency in z. The corresponding predicted gain lengths from GINGER simulations are $0.78 \mathrm{~m}$ and $0.44 \mathrm{~m}$, respectively.

The observed ratio of fundamental-to-second-harmonic intensities is $\sim 240$, similar to that as was seen for 8 March 2001. The predicted ratio at saturation is $\sim 1500$, again similar to 8 March 2001. The discrepancy is not fully understood but is at least partially due to the incomplete knowledge of the longitudinal variation of beam parameters, such as current, emittance, and the instantaneous energy spread, which can affect this ratio significantly.

\section{Conclusions}

In this paper we have presented experimental measurements of SASE emission from the APS SASE FEL at the LEUTL facility at both the fundamental and second harmonic. As expected, the second nonlinear harmonic emission exponentially grows and saturates near 
the same position in $\mathrm{z}$ as the fundamental. Numerical simulation results are in reasonable agreement with the measurements but are hampered by incomplete knowledge of the longitudinal profile of the electron beam properties. Beyond saturation, we found that both the fundamental and second harmonic emission are rich in phenomena.

\section{References}

[1] S.V. Milton et al., originally published in Science Express as 10.1126/science.1059955 on May 17, 2001 and Science 292 (2001) 2037.

[2] R. Bonifacio et al., Nucl. Instrum. Methods A 293 (1990) 627.

[3] H.P. Freund, S.G. Biedron, and S.V. Milton, IEEE J. Quantum Electron. 36 (2000) 275.

[4] Z. Huang and K.-J. Kim, Phys. Rev. E 62 (2000) 7295.

[5] Z. Huang and K.-J. Kim, Nucl. Instrum. Methods A 475 (2001) 112.

[6] G. Dattoli and P.L. Ottaviani, J. Appl. Phys. 86 (1991) 5331.

[7] L.-H. Yu, Phys. Rev. A 44 (1991) 5178.

[8] I. Ben-Zvi et al., Nucl. Instrum. Methods A 318 (1992) 208.

[9] L.-H. Yu et al., Science 289 (2000) 1932.

[10] R. Bonifacio et al., Nucl. Instrum. Methods A 296 (1990) 787.

[11] F. Ciocci et al., IEEE J. Quantum Electron. 31 (1995) 1242.

[12] W.M. Fawley et al., Proceedings of the IEEE 1995 Particle Accelerator Conference (1996) 219.

[13] H.P. Freund, S.G. Biedron, S.V. Milton, and H.-D. Nuhn, IEEE J. Quantum Electron., 37 (2001) 790.

[14] S.G. Biedron et al., Physics of, and Science with, the X-Ray Free-Electron Laser, 19th Advanced ICFA Beam Dynamics Workshop; Edited by M. Cornacchia, C. Pellegrini, S. Chattopadhyay, I. Lindau, August 2001, 0-7354-0022-9, CP 581, p. 203.

[15] S.G. Biedron et al., Proceedings of the 21st ICFA Beam Dynamics Workshop on LASER-BEAM INTERACTIONS, Stony Brook, USA, June 11-15, 2001 and submitted to Phys. Rev. Special Topics, Accelerators and Beams.

[16] A. Doyuran et al., Phys. Rev. Lett. 86 (2001) 5902-5905.

[17] G. Isoyama et al., Nucl Instrum and Meth in Phys Research A 475 (2001).

[18] R. Kato et al., "Higher Harmonic Generation Accompanied by SASE in the FarInfrared Region," these proceedings.

[19] A. Tremaine et al., "Experimental Characterization of SASE FEL Harmonic Radiation at Saturation," these proceedings.

[20] A.H. Lumpkin et al., "Comprehensive z-Dependent Measurements of Electron-Beam Microbunching Using COTR in a Saturated SASE FEL," these proceedings.

[21] V. Sajaev et al., "Z-dependent Spectral Measurements of SASE FEL at APS," these proceedings.

[22] W.M. Fawley, "An Informal Manual for GINGER and its post-processor XPLOTGIN,” LBID-2141, CBP Tech Note-104, UC-414, 1995.

[23] N.M. Kroll, P.L. Morton, and M.R. Rosenbluth, IEEE J. Quantum Electron. QE-17 (1981) 1436. 


\section{List of Tables:}

Table 1: Electron beam parameters for 8 March 2001.

Table 2: Electron beam measurements taken immediately after the 530-nm gain scan and the 265-nm gain scan, respectively, on 30 March 2001.

Table 3: Electron beam measurements taken immediately after the 530-nm and 265-nm UR gain scans on 16 August 2001. 
Table 1

\begin{tabular}{|l|c|}
\hline \multicolumn{1}{|c|}{ PARAMETER } & Value \\
\hline Beam energy $(\mathrm{MeV})$ & 217 \\
\hline Peak current $(\mathrm{A})$ & 260 \\
\hline Energy spread $(\%)$ & $0.1-0.2$ \\
\hline Bunch length $(\mathrm{fs} \mathrm{rms})$ & 250 \\
\hline Charge $(\mathrm{pC})$ & 160 \\
\hline Emittance $\mathrm{x}(\mu \mathrm{m})$ & 6.5 \\
\hline Emittance: $\mathrm{y}(\mu \mathrm{m})$ & 6.8 \\
\hline
\end{tabular}


Table 2

\begin{tabular}{|l|c|c|}
\hline \multicolumn{1}{|c|}{ PARAMETER } & 530-nm Gain Scan & 265-nm Gain Scan \\
\hline Beam energy $(\mathrm{MeV})$ & 217 & 217 \\
\hline Peak current $(\mathrm{A})$ & 505 & 360 \\
\hline Energy spread $(\%)$ & $0.1-0.2$ & $0.1-0.2$ \\
\hline Bunch length $(\mathrm{fs} \mathrm{rms})$ & 150 & 200 \\
\hline Charge $(\mathrm{pC})$ & 190 & 180 \\
\hline Emittance $\mathrm{x}(\boldsymbol{\mu m})$ & 9.0 & 6.5 \\
\hline Emittance: $\mathrm{y}(\boldsymbol{\mu m})$ & 8.0 & 7.1 \\
\hline
\end{tabular}


Table 3

\begin{tabular}{|l|c|}
\hline \multicolumn{1}{|c|}{ PARAMETER } & Value \\
\hline Beam energy $(\mathrm{MeV})$ & 217 \\
\hline Peak current $(\mathrm{A})$ & 210 \\
\hline Energy spread $(\%)$ & $0.1-0.2$ \\
\hline Bunch length $(\mathrm{fs} \mathrm{rms})$ & 340 \\
\hline Charge $(\mathrm{pC})$ & 176 \\
\hline Emittance $\mathrm{x}(\mu \mathrm{m})$ & 5.9 \\
\hline Emittance: $\mathrm{y}(\mu \mathrm{m})$ & 6.4 \\
\hline
\end{tabular}




\section{List of Figures:}

Figure 1: Measured fundamental and second harmonic UR energy from 8 March 2001 compared with the simulated and analytical counterparts.

Figure 2: Three measured second harmonic, single-shot spectra using the end-station spectrometer for each undulator/station: (a), (b), (c), and (d), from 8 March 2001.

Figure 3: The average rms spectral width of the second harmonic emission after stations 5 $(12 \mathrm{~m}), 6(14.4 \mathrm{~m}), 7(16.8 \mathrm{~m})$, and $8(19.2 \mathrm{~m})$ as a function of distance.

Figure 4: Measured fundamental energy from 30 March 2001 compared with the simulated counterpart.

Figure 5: Measured second harmonic UR energy from 30 March 2001 compared with the analytical counterpart.

Figure 6: Simultaneous fundamental and second harmonic spectra after undulator 7 ( $\mathrm{z}=$ $16.8 \mathrm{~m}$ ) as measured by the end-station spectrometer.

Figure 7: Measured fundamental and second harmonic UR energy from 16 August 2001 compared with the simulated and analytical counterparts. 


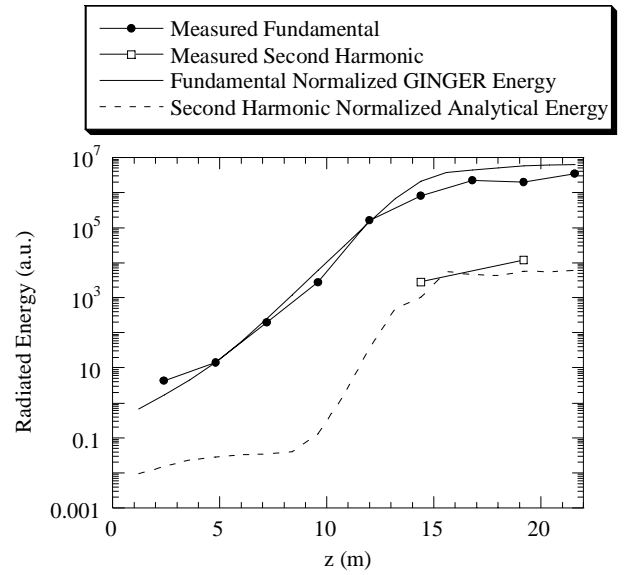

Figure 1 


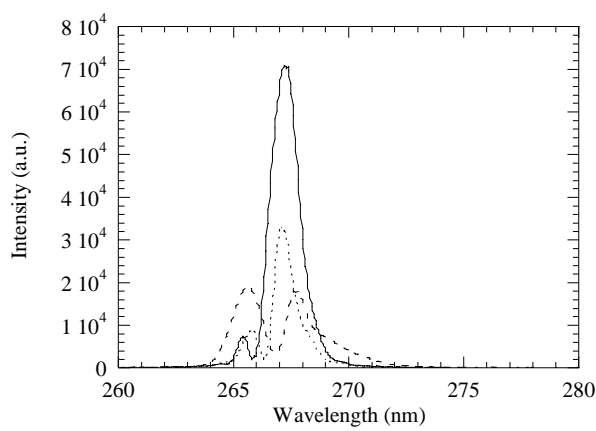

(a) Station 5 (12 m)

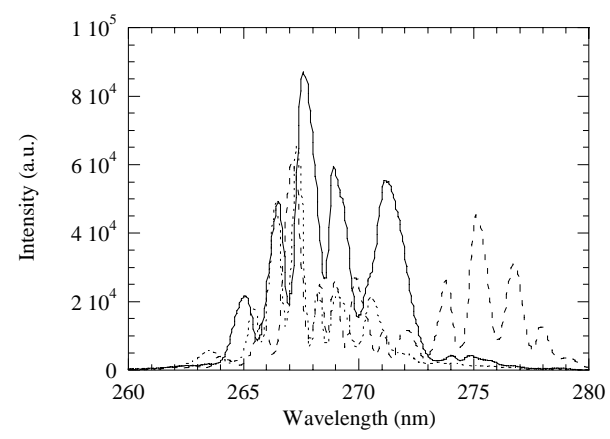

(c) Station 7 (16.8 m)

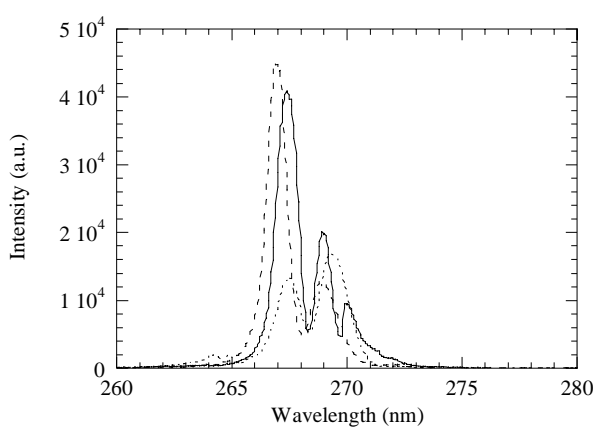

(b) Station 6 (14.4 m)

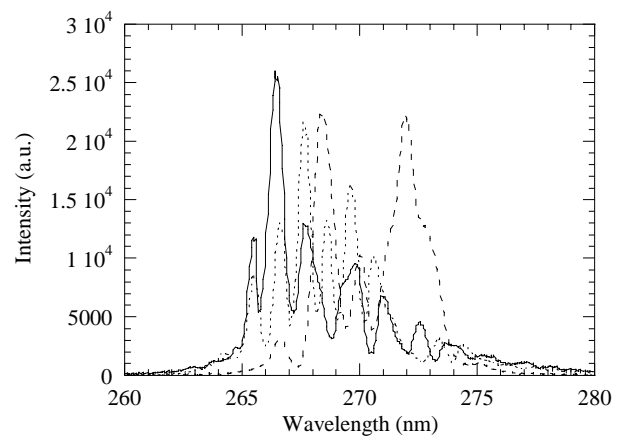

(d) Station 8 (19.2 m)

Figure 2 


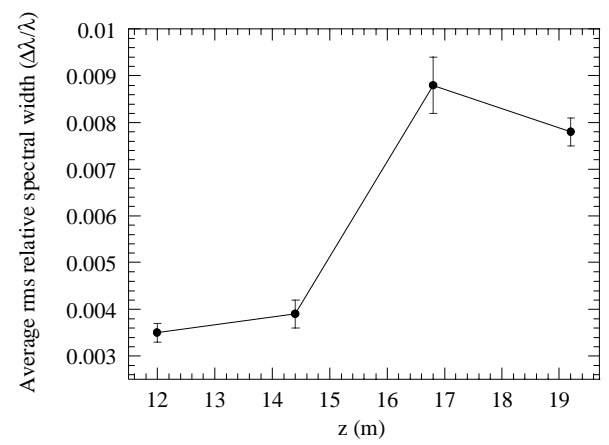

Figure 3 


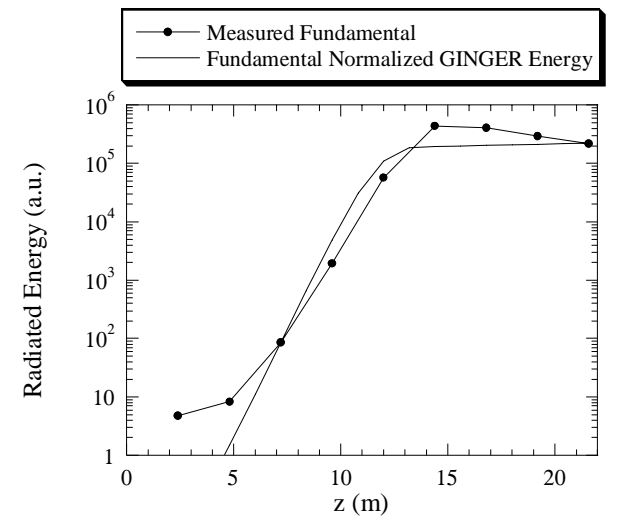

Figure 4 


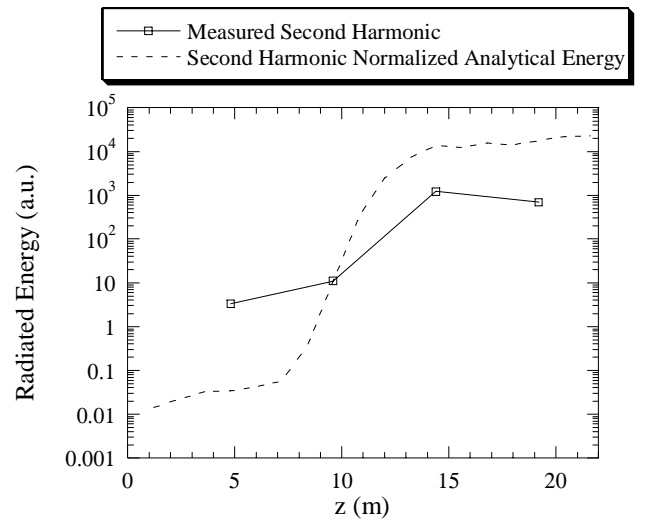

Figure 5 


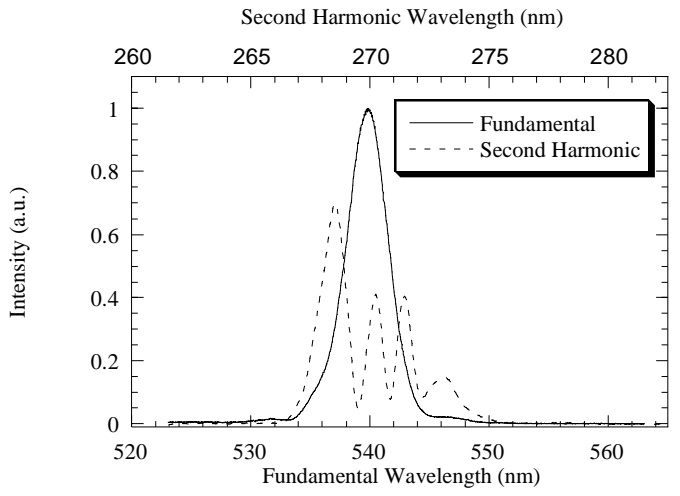

Figure 6 


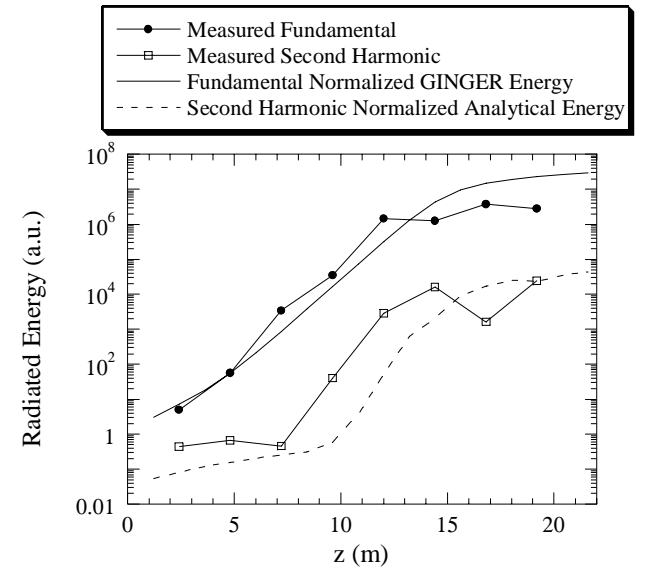

Figure 7 